\title{
RNAi knockdown of Hop (Hsp70/Hsp90 organising protein) decreases invasion via MMP-2 down regulation
}

\author{
Naomi Walsh ${ }^{\mathrm{a}, *}$, AnneMarie Larkin ${ }^{\mathrm{a}}$, Niall Swan ${ }^{\mathrm{c}, 1}$, Kevin Conlon ${ }^{\mathrm{c}}$, Paul Dowling ${ }^{\mathrm{a}}$, \\ Ray McDermott ${ }^{\mathrm{c}}$, Martin Clynes ${ }^{\mathrm{a}, \mathrm{b}}$ \\ ${ }^{a}$ National Institute for Cellular Biotechnology, Dublin City University, Glasnevin, Dublin 9, Ireland \\ ${ }^{\mathrm{b}}$ Molecular Therapeutics for Cancer Ireland, Dublin City University, Glasnevin, Dublin 9, Ireland \\ ' The Centre for Pancreaticobiliary Disease, The Adelaide and Meath Hospital, Dublin incorporating the National Children's Hospital, Tallaght, Dublin 24, Ireland
}

\section{A R T I C L E I N F O}

\section{Article history:}

Received 27 January 2011

Received in revised form 9 March 2011

Accepted 11 March 2011

\section{Keywords:}

Hop/STIP1/STI1 (Hsp70/Hsp90-organising

protein)

Pancreatic cancer

Immunohistochemistry

In vitro invasion

MMP-2

\begin{abstract}
A B S T R A C T
We previously identified Hop as over expressed in invasive pancreatic cancer cell lines and malignant tissues of pancreatic cancer patients, suggesting an important role for Hop in the biology of invasive pancreatic cancer. Hop is a co-chaperone protein that binds to both Hsp70/Hsp90. We hypothesised that by targeting Hop, signalling pathways modulating invasion and client protein stabilisation involving Hsp90-dependent complexes may be altered.

In this study, we show that Hop knockdown by small interfering (si)RNA reduces the invasion of pancreatic cancer cells, resulting in decreased expression of the downstream target gene, matrix metalloproteinases-2 (MMP-2). Hop in conditioned media co-immunoprecipitates with MMP-2, implicating a possible extracellular function for Hop. Knockdown of Hop expression also reduced expression levels of Hsp90 client proteins, HER2, Bcr-Abl, c-MET and $\mathrm{v}$-Src. Furthermore, Hop is strongly expressed in high grade PanINs compared to lower PanIN grades, displaying differential localisation in invasive ductal pancreatic cancer, indicating that the localisation of Hop is an important factor in pancreatic tumours.

Our data suggests that the attenuation of Hop expression inactivates key signal transduction proteins which may decrease the invasiveness of pancreatic cancer cells possibly through the modulation of Hsp90 activity. Therefore, targeting Hop in pancreatic cancer may constitute a viable strategy for targeted cancer therapy.
\end{abstract}

(c) 2011 Elsevier Ireland Ltd. All rights reserved.

\section{Introduction}

Pancreatic adenocarcinoma is one of the most lethal and poorly understood human malignancies. The 5-year

Abbreviations: PanIN, pancreatic intraepithelial neoplasia; IHC, immunohistochemistry; 17-AAG, 17-allylamino-17 demethoxygeldanamycin.

* Corresponding author. Tel.: +353 (0) 17006263; fax: +353 (0) 17005484.

E-mail addresses: Naomi.walsh@dcu.ie (N. Walsh), Annemarie.larkin @dcu.ie (A. Larkin), N.Swan@st-vincents.ie (N. Swan), Kevin.Conlon@ amnch.ie (K. Conlon), paul.dowling@dcu.ie (P. Dowling), Ray.McDermott @amnch.ie (R. McDermott), martin.clynes@dcu.ie (M. Clynes).

1 Current address: Dept of Histopathology, St. Vincent's University Hospital, Elm Park, Dublin 4, Ireland. survival rate is between $1 \%$ and $4 \%$ [1], mainly due to the lack of effective therapies. Conventional treatment used to treat pancreatic cancer such as surgery, chemotherapy, radiation or combinations of these have had very little effect on the overall survival of this disease [2].

Hop (Hsp70/Hsp90-organising protein) or STIP1 (stress-induced phosphoprotein 1) mediates the association, and forms a physical complex with the molecular chaperones heat shock proteins (Hsp) Hsp70 and Hsp90 $[3,4]$ which is dependent on the hydrolysis of ATP and ADP/ATP exchange. Hop optimises the functional cooperation between Hsp70 and Hsp90 which requires highly co-ordinated interactions for the folding and conformational regulation of a variety of proteins [5]. The 
molecular chaperone complex of Hop, Hsp70 and Hsp90 is involved in the folding and maturation of key regulatory proteins involved in cell viability [6], such as steroid hormone receptors, transcription factors and kinases (some of which are involved in cancer progression). The role of Hop in tumour progression is not fully elucidated; however, Hop has been shown to be over expressed in colonic carcinoma cells [7] and in hepatocellular carcinoma (HCC) [8]. Hop is secreted by and shown to induce proliferation in glioma tumour cells through MAPK and P13K pathways [9]. Hop has also been implicated in the controlled death process. Bredemeyer et al. [10] confirmed Hop as a substrate of the apoptotic protease granzyme B (GzmB), as Hop was directly cleaved by GzmB in vitro and in cells undergoing GzmB-induced apoptosis. Expression of the two cleavage fragments of Hop did not induce cell death; however, cleavage of Hop by GzmB did result in loss of Hop function in vitro.

There is increasing evidence that heat shock proteins play an important role in the development, maintenance and progression of cancer [11]. Heat shock proteins such as, Hsp90 and Hsp70 have previously been implicated in pancreatic cancer $[12,13]$. Hsps are highly conserved molecules, which protect cells from various environmental damages, including stress and carcinogenesis [14] and are activated by the ubiquitin-proteasome pathway. The covalent attachment of ubiquitin to protein regulates a number of cellular processes including responses to stress. This process is responsible for selective targeting of proteins for degradation. The induced Hsps play roles as cellular chaperones, and many modulate apoptotic pathways, particularly those involving mitochondria, conferring protection from stressful stimuli including chemotherapeutic agents [15]. Hsp90 client proteins include mutated p53, Bcr-Abl, Raf-1, Akt, HER2 (ErbB2) and hypoxia inducible factor-1alpha (HIF-1alpha) [16].

We previously profiled Hop expression in an in vitro pancreatic cancer cell line invasion model and pancreatic tumour tissue specimens [17]. In the present study, we hypothesised that modulation of Hop expression could alter invasion and affect the activity of Hsp90 and its client proteins; suppression of Hop by siRNA reduced invasion through decreased expression of MMP-2. We further show that modulation of Hop alters the activities of representative Hsp90 client proteins HER2, Bcr-Abl, c-MET and v-Src. This study reports that Hop is likely to play a role in invasion of pancreatic cancer cells, and possibly contribute to the highly aggressive metastatic phenotype of pancreatic cancer. In addition our findings support Hop as a potential therapeutic target for the treatment of pancreatic cancer.

\section{Materials and methods}

\subsection{Cell lines}

The human pancreatic cell lines Panc-1, SW1990, Capan-1, MiaPaCa-2 (ATCC, USA), HPAC (DSMZ, Germany) and BxPc-3 (ECACC, UK) were used in this study. Clone \#3 and Clone \#8 were previously isolated by single cell dilution from MiaPaCa-2 in this laboratory [18]. Cells were maintained in a humidified atmosphere containing $5 \% \mathrm{CO}_{2}$ at $37^{\circ} \mathrm{C}$ in Dulbecco's modified Eagles medium (DMEM) supplemented with $5 \%(\mathrm{v} / \mathrm{v})$ fetal bovine serum (Sigma-Aldrich). All cell lines were free from Mycoplasma as tested with the indirect Hoechst staining method.

\subsection{Invasion assays}

Invasion assays were performed as previously described [19]. Briefly, matrigel was diluted to $1 \mathrm{mg} / \mathrm{ml}$ in serum-free DMEM. $100 \mu \mathrm{l}$ of matrigel was coated on each Boyden chamber insert (Falcon) $(8.0 \mu \mathrm{m}$ pore size), in a 24 -well plate (Costar) and incubated overnight at $4{ }^{\circ} \mathrm{C}$. The matrigel was allowed polymerise at $37^{\circ} \mathrm{C}$ for $1 \mathrm{~h}$, then washed with DMEM. $100 \mu \mathrm{l}$ of complete DMEM was added to the wells and $1 \times 10^{5} / 100 \mu$ l cells were then seeded onto the insert. An aliquot of $500 \mu \mathrm{l}$ of complete DMEM was added into the underside of the well. After $24 \mathrm{~h}$ incubation, the inside of the insert was wiped with a wet cotton swab. The under surface was gently rinsed with PBS and stained with $0.25 \%(\mathrm{w} / \mathrm{v})$ crystal violet for $10 \mathrm{~min}$, rinsed again with sterile water and allowed to dry. The inserts were then viewed under the microscope and the number of cells per field in 10 random fields, were counted at $200 \times$ magnification. The average number of cells per field was then multiplied by a factor of 140 (growth area of membrane/ field area viewed at $200 \times$ magnification (calibrated using a microscope graticule)) to determine the total number of invading cells. Experiments were performed in triplicate.

\subsection{Immunoblotting}

Whole protein was extracted from cell lysates using $1 \times$ lysis buffer ( $50 \mathrm{mM}$ Tris-Cl, $150 \mathrm{mM} \mathrm{NaCl}$, and $0.5 \%(\mathrm{v} / \mathrm{v})$ $\mathrm{NP}-40$ ). Lysates were centrifuged for $15 \mathrm{~min}$ at $16,000 \mathrm{~g}$ at $4{ }^{\circ} \mathrm{C}$. Protein concentrations were determined using the Bio-Rad Bradford protein assay according to manufacturer's instructions (Bio-Rad). A concentration of $25 \mu \mathrm{g}$ of protein was separated by 7.5 and $10 \%(\mathrm{w} / \mathrm{v})$ SDS-PAGE gels under reducing conditions. Proteins were transferred to nitrocellulose membrane (Amersham). Membranes were blocked at $4{ }^{\circ} \mathrm{C}$ overnight in TBS ( $25 \mathrm{mM}$ Tris- $\mathrm{HCl}, \mathrm{pH} 7.4$, $150 \mathrm{mM} \mathrm{NaCl}, 2.7 \mathrm{mM} \mathrm{KCl}$ ) containing 5\% (w/v) lowfat milk powder. Membranes were probed with antibodies Hop/STIP1 (ab56873, Abcam), Hsp70, Hsp90 (\#4872, \#4875, Cell Signaling), Bcr-Abl (\#2862, Cell Signaling), HER2 (\#2165, Cell Signaling), c-MET (sc-10, Santa Cruz), v-Src (OP07T, Merck) and $\beta$-actin (Sigma). Membranes were washed $3 \times$ for 5 min with PBS-Tween-20 $(0.5 \% \mathrm{v} / \mathrm{v})$ and incubated with secondary antibodies, anti-mouse (Sig$\mathrm{ma}$ ) and anti-rabbit (Cell Signaling) for $1 \mathrm{~h}$ at RT and washing step repeated. Detection was performed with Luminol reagent (Santa Cruz Biotechnology).

\section{4. siRNA transient transfections}

Three pre-designed siRNAs (Ambion) were chosen for each of the protein/gene targets and transfected into cells. Hop/STIP1 siRNA targets (Ambion, \#106197, \#106196, and \#106195). For each set of siRNA transfections carried out, a 
control (non-transfected) and a scrambled (SCR) (Ambion, \# 17010) were used. siRNA experiments were set up using $2 \mu \mathrm{l} \mathrm{NeoFx}$ (Ambion, AM4511), to transfect $30 \mathrm{nM}$ siRNA at a cell density of $3 \times 10^{5} / \mathrm{ml}$ in a 6 -well plate. Transfection medium was removed after $24 \mathrm{~h}$ and replaced with fresh growth medium. The transfected cells were collected at 72 and $96 \mathrm{~h}$ for immunoblotting and assayed for changes in invasion capacity at $72 \mathrm{~h}$ using the in vitro invasion assay (as previously described).

\subsection{Zymography}

Pancreatic cancer cells ( $\sim 70-80 \%$ confluent) were washed and replenished with serum-free DMEM for $48 \mathrm{~h}$. Cells were then pretreated with various concentrations of 17-AAG (Merck) for $24 \mathrm{~h}$ vs control conditioned media in serum-free conditions for $72 \mathrm{~h}$. The volume of conditioned medium was normalised by cell number. CM samples were denatured, run on $10 \%(\mathrm{w} / \mathrm{v})$ gelatin gels (Invitrogen Life Technologies) and renatured in zymogram renaturing buffer and developed in zymogram developing buffer (Invitrogen). Gels were stained with $0.5 \%(\mathrm{w} / \mathrm{v})$ coomassie blue, destained in a solution containing $10 \%(\mathrm{v} / \mathrm{v})$ methanol and $7.5 \%(\mathrm{v} / \mathrm{v})$ acetic acid. Zones of gelatinolytic activity were detected as clear bands against a blue background.

\subsection{Immunoprecipitation (IP)}

Protein from conditioned media was lysed in buffer (4\% w/v CHAPS, $7 \mathrm{M}$ urea, $2 \mathrm{M}$ thiourea, $10 \mathrm{mM}$ Tris$\mathrm{HCl}, 5 \mathrm{mM}$ magnesium acetate $\mathrm{pH} 8.5$ ) and incubated with $200 \mu \mathrm{g}$ of Hop (sc-136082, Santa Cruz) or control mouse $\operatorname{IgG}$, at $4{ }^{\circ} \mathrm{C}$, shaking, for $30 \mathrm{~min}$. In order to precipitate the antibody/antigen complex, $50 \mu \mathrm{l}$ of packed Protein-L agarose beads (\#20512, Pierce) were added and samples incubated overnight on a rocking platform at $4{ }^{\circ} \mathrm{C}$. Samples were then centrifuged at $16,000 \mathrm{~g}$ for $25 \mathrm{~s}$ and pellets washed with IP wash buffer $3 \times$. Samples were centrifuged gently after each wash step. Supernatants were removed and pellets resuspended in $50 \mu \mathrm{l}$ denaturing buffer and boiled at $95{ }^{\circ} \mathrm{C}$ for $5 \mathrm{~min}$. Denatured samples were then centrifuged at $16,000 \mathrm{~g}$ for $3 \mathrm{~min}$ and supernatant collected and stored at $-20^{\circ} \mathrm{C}$. Immunoprecipitates were separated on $7.5 \%(\mathrm{w} / \mathrm{v})$ SDS PAGE gels and probed with Hsp90 (\#4875, Cell Signaling), Hop/STIP1 (ab56873, Abcam) and MMP-2 (IM53, Merck) antibodies.

\subsection{Immunohistochemistry}

Formalin-fixed paraffin-embedded pancreatic tumour $4-\mu \mathrm{m}$ sections of tissue blocks were cut using a microtome, mounted onto poly-l-lysine coated slides and dried overnight at $37^{\circ} \mathrm{C}$. Slides were stored at room temperature until required. Briefly, the slides were immunohistochemically stained using primary antibodies specific for Hop/STIP1 (ab56873, Abcam). After deparaffinisation and rehydration, the slides were subjected to an antigen retrieval step consisting of 20-min incubation in $\mathrm{pH} 9.0$ buffer (Target Retrieval, Dako) in a $95^{\circ} \mathrm{C}$ water bath followed by cooling to room temperature. Staining was performed using an automated staining apparatus for IHC (Autostainer, Dako) according to the manufacturer's guidelines. The proper positive and negative controls were processed at the same time. The slides were counterstained with haematoxylin, dehydrated in graded alcohols and mounted (DPX, Sigma). Scoring and interpretation was carried out by consultant pathologist NS.

\subsection{Statistical analysis}

Student's $t$-tests (two-tailed, two-sample unequal variance) were used to analyse differences in protein expression between comparisons. A $p$-value $\leqslant 0.05^{*}, \leqslant 0.01^{* *}$ and $\leqslant 0.005^{* * *}$ was considered statistically significant.

\section{Results}

3.1. Hop is associated with invasive pancreatic carcinoma

We previously identified Hop/STIP1 by 2D DIGE followed by MALDITOF MS as 2-fold more abundant in Clone \#3, a highly invasive sub-population of the human pancreatic cancer cell line MiaPaCa-2, compared to the low invasive Clone \#8 [17]. In this study, we examined constitutive Hop expression in a panel of seven human pancreatic cancer cell lines by Western immunoblot (Fig. 1A) and studied its expression with the invasive status of the cell lines (Fig. 1B and C). All cell lines express Hop but higher abundance of Hop was observed in the more invasive cell lines.

\subsection{Hop, as a novel inducer of pancreatic cancer cell invasion}

To determine whether loss of Hop affects the ability of cells to invade in vitro, we compared the ability of BxPc-3 and Panc-1 (high Hop expression) human pancreatic cancer cell lines to invade through matrigelcoated Boyden chambers post Hop-siRNA transfection. As Hop-siRNA (2) and (3) were more effective at Hop knockdown (Fig. 5), we used these siRNAs for further studies. Invasion of Panc- 1 cells was reduced by $50 \%$ $(p=0.0005)$ using Hop-siRNA ( 2$)$ and by $50 \%(p=0.003)$ with Hop-siRNA (3) (Fig. 2A). Invasion of BxPc-3 transfected cells were reduced by $51 \%$ $(p=0.002)$ using Hop-siRNA $(2)$ and by $46 \%(p=0.002)$ in the case of Hop-siRNA (3) (Fig. 2B).

\subsection{Matrix metalloproteinases down regulated as a result of Hop knockdown}

We further investigated how knockdown of Hop using siRNA could affect invasion. Previously, secretion of HSP90 $\alpha$ into the extracellular matrix surrounding tumour cells was reported to assist the activation of MMP-2 as well as contributing to tumour cell invasiveness [20,21]. We show that Hop is found in the conditioned medium of both Panc- 1 and BxPc-3 cells and therefore may possibly have an extracellular role in Hsp90 client protein activation (Fig. 3A). Inhibition of Hop by Hop-siRNA transfection reduced the expression of MMP-2 in both Panc-1 and BxPc-3 as determined by immunoblotting (Fig. 3B).

\subsection{Hop co-immunoprecipitates with MMP-2}

Our findings suggest that Hop may act as an extracellular protein; we confirmed that Hop co-immunoprecipitates with MMP-2 in BxPc-3 conditioned media, showing a direct interaction between these proteins (Fig. 4A), suggesting that Hop may be acting as an extracellular cochaperone to Hsp90, in turn activating MMP-2. Our studies support this suggestion as Hop, p23 and Hsp90 were found in the conditioned media of HT-1080 cells, showing a direct interaction between these proteins extracellularly [21]. Inhibition of Hop may have affected MMP-2 expression and activity through altering the activity of extracellular Hsp90. A study by Tsutsumi et al. [22] using the non cell-permeable Hsp90 inhibitor, DMAG-N-oxide, reduced in vitro tumour cell 
A
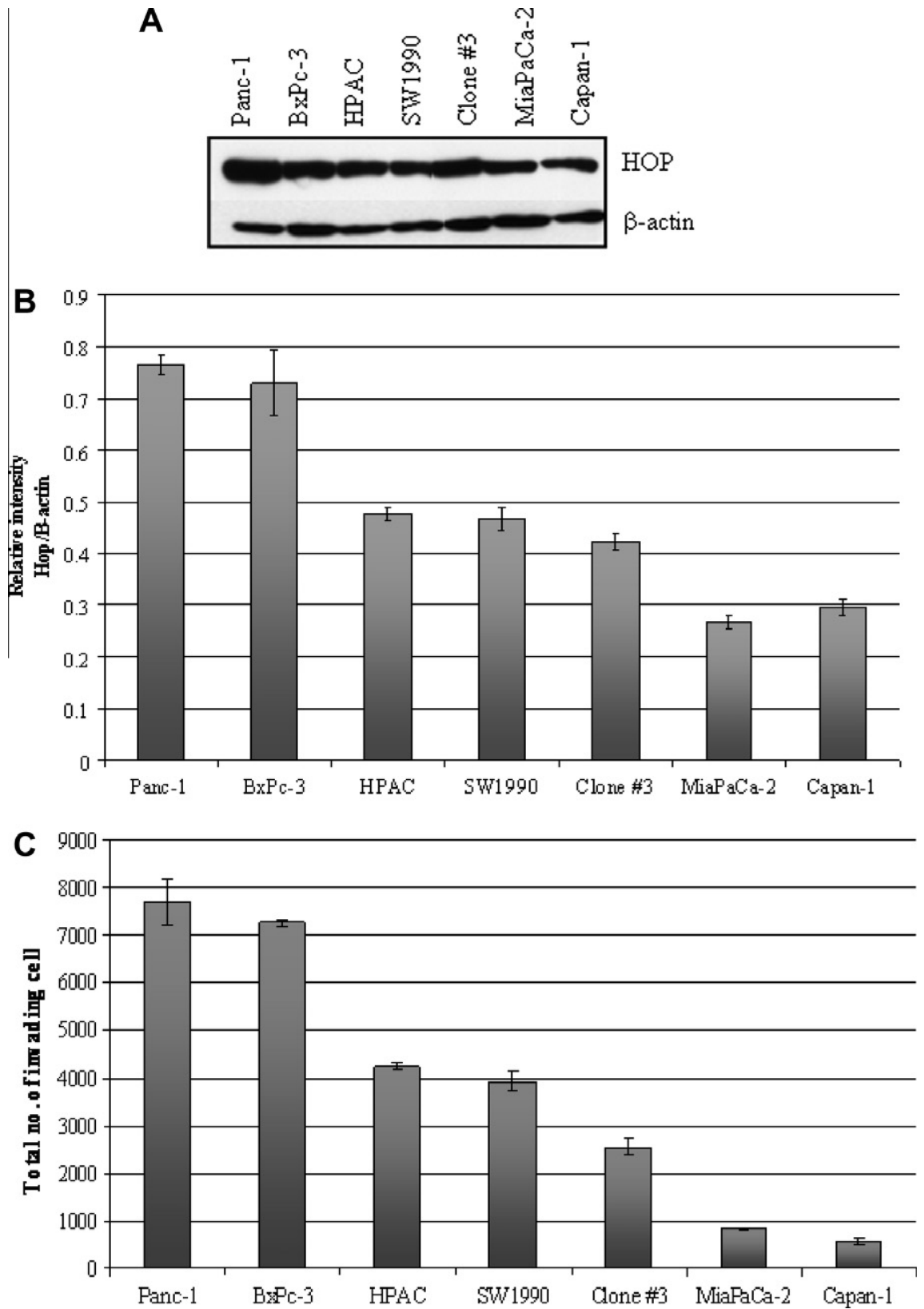

Fig. 1. Hop protein expression in human pancreatic cancer cells. (A) Representative Western immunoblot showing the expression of constitutive Hop in a panel of pancreatic cancer cell lines. Housekeeping gene $\beta$-actin was used as an internal control. (B) Densitometry analysis of Hop bands (normalised to $\beta$ actin). Error bars represent the standard deviation of triplicate experiments. (C) The invasive capacity of panel of cell lines. The total number of invading cells was determined by counting the number of cells per field in 10 random fields, at $200 \times$ magnification. The average number of cells per field was then multiplied by a factor of 140 (growth area of membrane/field area viewed at $200 \times$ magnification (calibrated using a microscope graticule)). Error bars represent the standard deviation of triplicate invasion assay experiments $(n=3)$.

invasion and migration and decreased melanoma lung metastasis in vivo. The monoclonal antibody $(\mathrm{mAb})$ directed against Hsp90, mAb $4 \mathrm{C} 5$, inhibited melanoma cell invasion and significantly reduced metastasis in vivo by binding to surface Hsp90 as it is not internalised, further indicating an extracellular role for Hsp90 [23]. We also show that the specific Hsp90 inhibitor, geldanamycin (17-AAG) at $0.1 \mu \mathrm{M}$ and $1 \mu \mathrm{M}$ blocks MMP-2 and MMP-9 [24] activation as revealed by zymography (Fig. 4B). Invasion in the highly invasive Panc-1 and BxPc-3 cells lines was also reduced at $1 \mu \mathrm{M}$ 17-AAG (Fig. 4C and D), proliferation and cell survival at this concentration was not effected (data not shown).
3.5. Role of Hop as an Hsp90/Hsp70 co-chaperone protein - silencing of Hop alters expression of downstream Hsp90 client proteins

Hsp90 client proteins play important roles in the regulation of the cell cycle, cell growth, cell survival, apoptosis, and tumour progression. To investigate whether Hop could serve as a therapeutic target in pancreatic cancer, we used Hop-siRNA to knockdown Hop expression in three invasive human pancreatic cancer cell lines: Clone \#3, BxPc-3 and Panc-1. $72 \mathrm{~h}$ post Hop-siRNA transfection, Hop expression was reduced in Clone \#3, BxPc-3 and Panc-1 (Fig. 5). Hop-siRNA did not alter levels of Hsp70 or Hsp90 expression in BxPc-3, Panc-1 and 


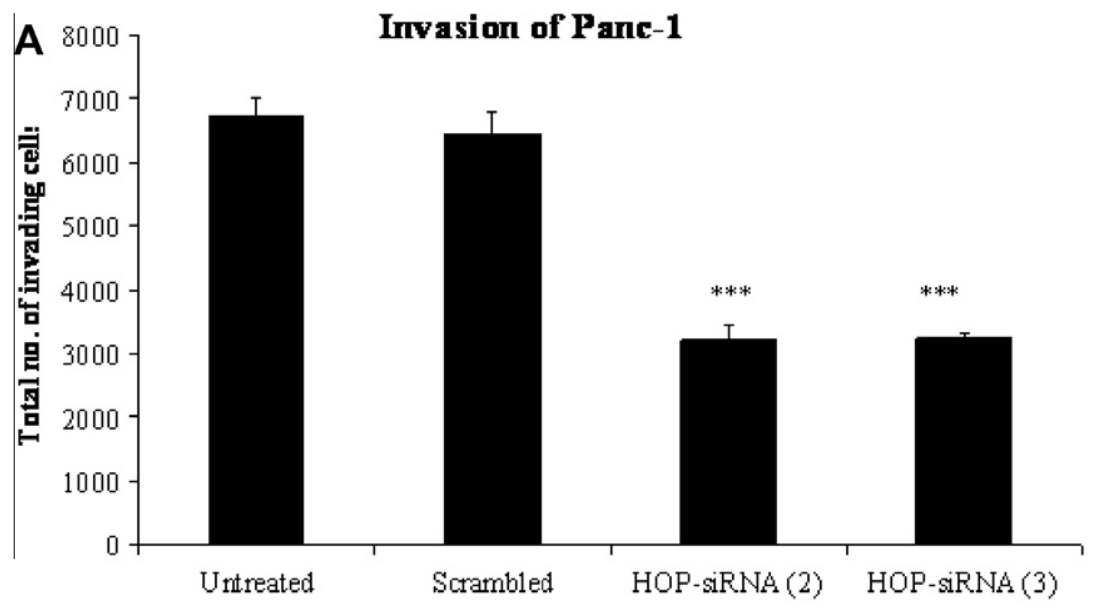

B

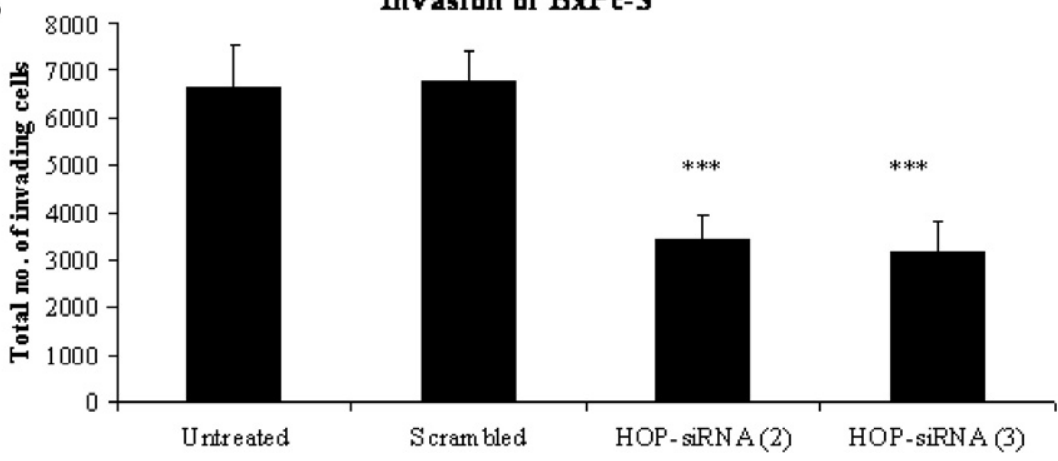

Fig. 2. Knockdown of Hop reduces the invasion of Panc- 1 and BxPc-3 cells. A. Panc- 1 and B. BxPc-3 cells were transfected with non-targeting scrambled siRNA (control siRNA) and two independent siRNA sequences against Hop. The total number of invading cells was determined by counting the number of cells per field in 10 random fields, at $200 \times$ magnification. The average number of cells per field was then multiplied by a factor of 140 (growth area of membrane/field area viewed at $200 \times$ magnification (calibrated using a microscope graticule)). Error bars represent the standard deviation of triplicate invasion assay experiments $(n=3)$.

A

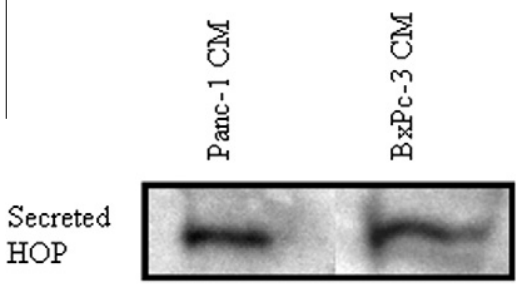

B

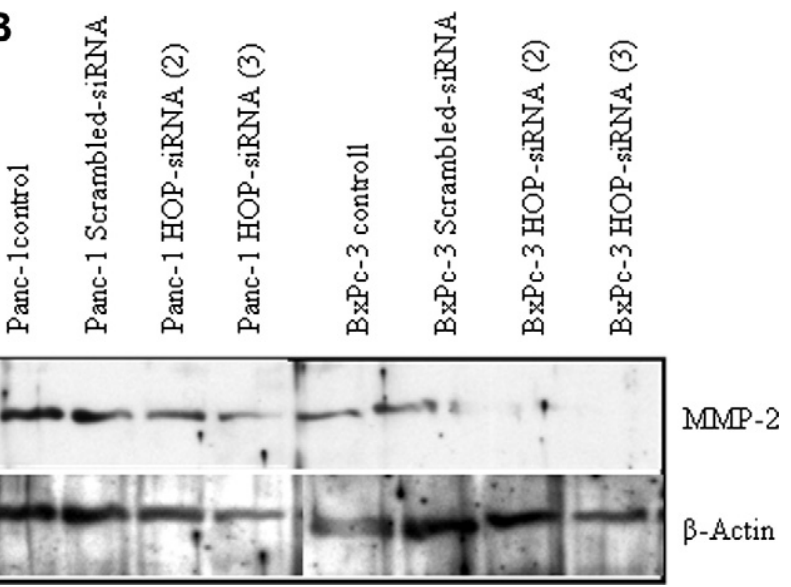

Fig. 3. Hop as an extracellular protein can disrupt MMP-2 expression. (A) Serum-free conditioned media from Panc-1 and BxPc-3 cells was collected, concentrated and immunoblotted for Hop. (B) Panc-1 and BxPc-3 cells transfected with Hop-siRNA (2) and (3) were immunoblotted for MMP-2.

Clone \#3 cell lines; however, the expression of the Hsp90 client proteins, HER2, Bcr-Abl, c-MET and v-Src were reduced with Hop-siRNA transfection (Fig. 5). These results suggest that the effect of Hop knockdown in pancreatic cancer may be mediated through these proteins.
3.6. Localisation of Hop membrane staining in pancreatic cancer tumours

In a small panel of pancreatic tumours we identified cytoplasmic Hop expression in areas of tumour invasion and in necrotic cells in the epithelium of paraffin-embedded pancreatic tumour specimens. 
A

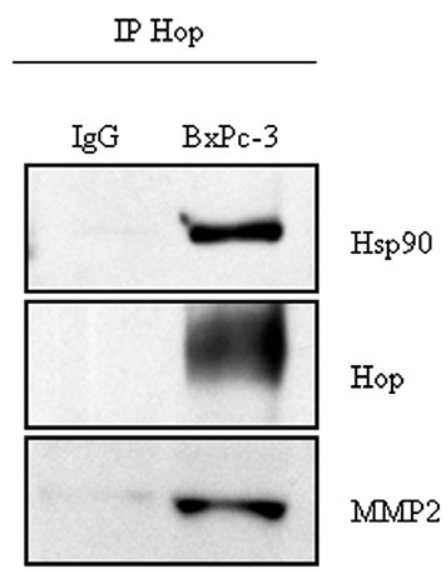

B

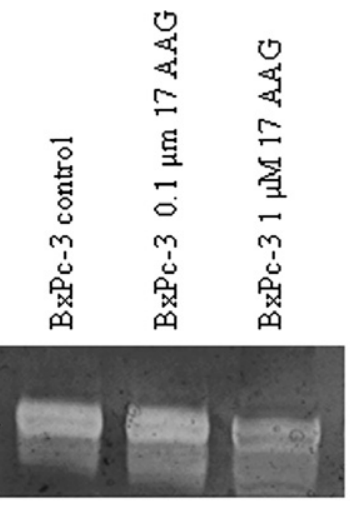

72

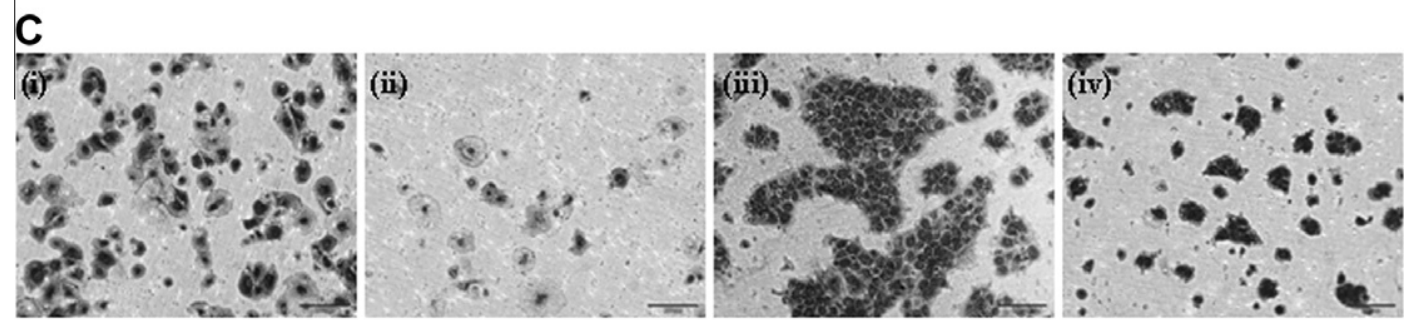

Invas inn ass ay
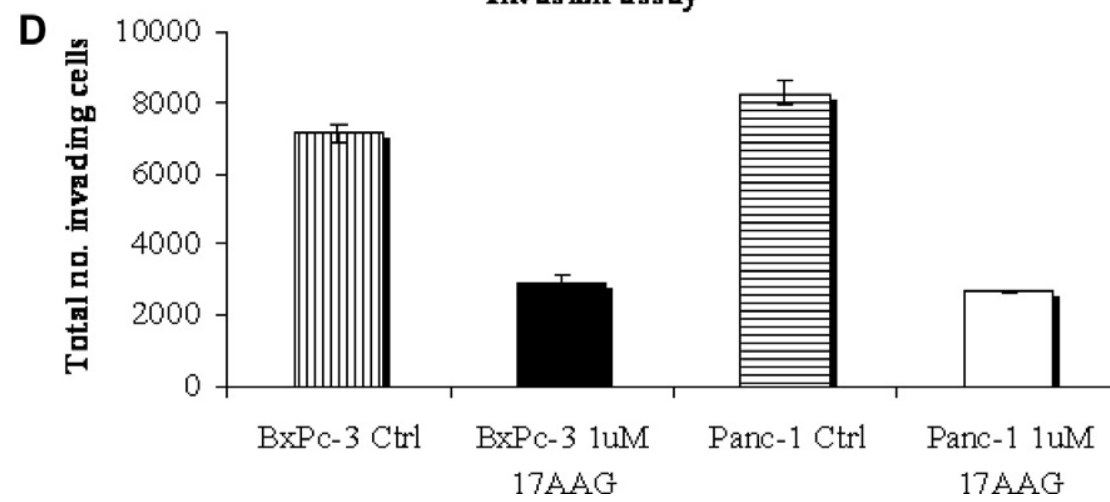

Panc-1 Ctrl

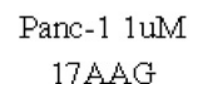

Fig. 4. Hop co-immunoprecipitates with Hsp90 and MMP-2. (A) Hop was immunoprecipitated from BxPc-3 conditioned media as described in materials and methods. Proteins were detected by MMP-2 and Hsp90 antibodies as indicated (right side of panel). (B) Effect of 17-AAG on pancreatic cancer. To validate Hop as an inhibitor of invasion in the Hsp90-complex, we used the Hsp90 pharmacological inhibitor 17-AAG and observed its effect on MMP-2 and MMP-9 expression. BxPc-3 cells were treated with $0.1 \mu \mathrm{M}$ and $1 \mu \mathrm{M}$ of $17-\mathrm{AAG}$ for $24 \mathrm{hrs}$ under serum-free conditions. Conditioned medium was collected and MMP-2 and MMP-9 activities were analysed by gelatin zymography. (C) Representative invasion assay pictures of cells invaded through Boyden invasion chambers $(8 \mu \mathrm{m}$ pore size) coated with matrigel basement membrane matrix stained with $0.25 \%(\mathrm{w} / \mathrm{v})$ crystal violet under control conditions and $24 \mathrm{~h}$ treatment with $1 \mu \mathrm{M}$ 17-AAG in Panc- 1 and BxPc-3 cells. Magnification $200 \times$, scale bar $200 \mu \mathrm{m}$. (D) Invasion potential of Panc-1 and BxPc-3 cells treated with $1 \mu \mathrm{M}$ 17-AAG as outlined in materials and methods. Graphs represented as the total number of invading cells, as determined by counting the number of cells per field in 10 random fields, at $200 \times$ magnification. The average number of cells per field was then multiplied by a factor of 140 (growth area of membrane/field area viewed at $200 \times$ magnification (calibrated using a microscope graticule)). Values are mean \pm standard deviation $(n=3$ ).

Cytoplasmic Hop staining was also observed in the normal ductal and acinar cells of the exocrine pancreas but at a lesser intensity compared to tumour [17]. To explore Hop as a potential target for invasive pancreatic cancer, we studied the profile of 17 pancreatic tumours, 15 invasive/metastatic pancreatic ductal adenocarcinomas (PDACs), 1 cholangiocarcinoma and 1 intraductal papillary mucinous neoplasm (IPMN). All PDACs specimens displayed cytoplasmic Hop staining (Fig. 6A and B); in general stronger Hop staining was observed in higher PanIN than in lower PanIN grades. Fig. 6A shows strong Hop staining in PanIN grade 3 (arrow 1) compared to negligible staining in PanIN grade1 (arrow 2). The invasive front of the tumour also displayed strong Hop staining as shown in Fig. 6B by perineural invasion. The nerve (arrow 1) is infiltrated by invasive pancreatic cells with high Hop expression (arrow 2). We also observed differential localised (subnuclear and membrane) staining of Hop. In Fig. 6C, linear sub-nuclear staining of Hop was seen in a PanIN grade1 tumour. Furthermore, membrane localisation of Hop was observed in an invasive pancreatic tumour with mucinous features (Fig. 6D). 


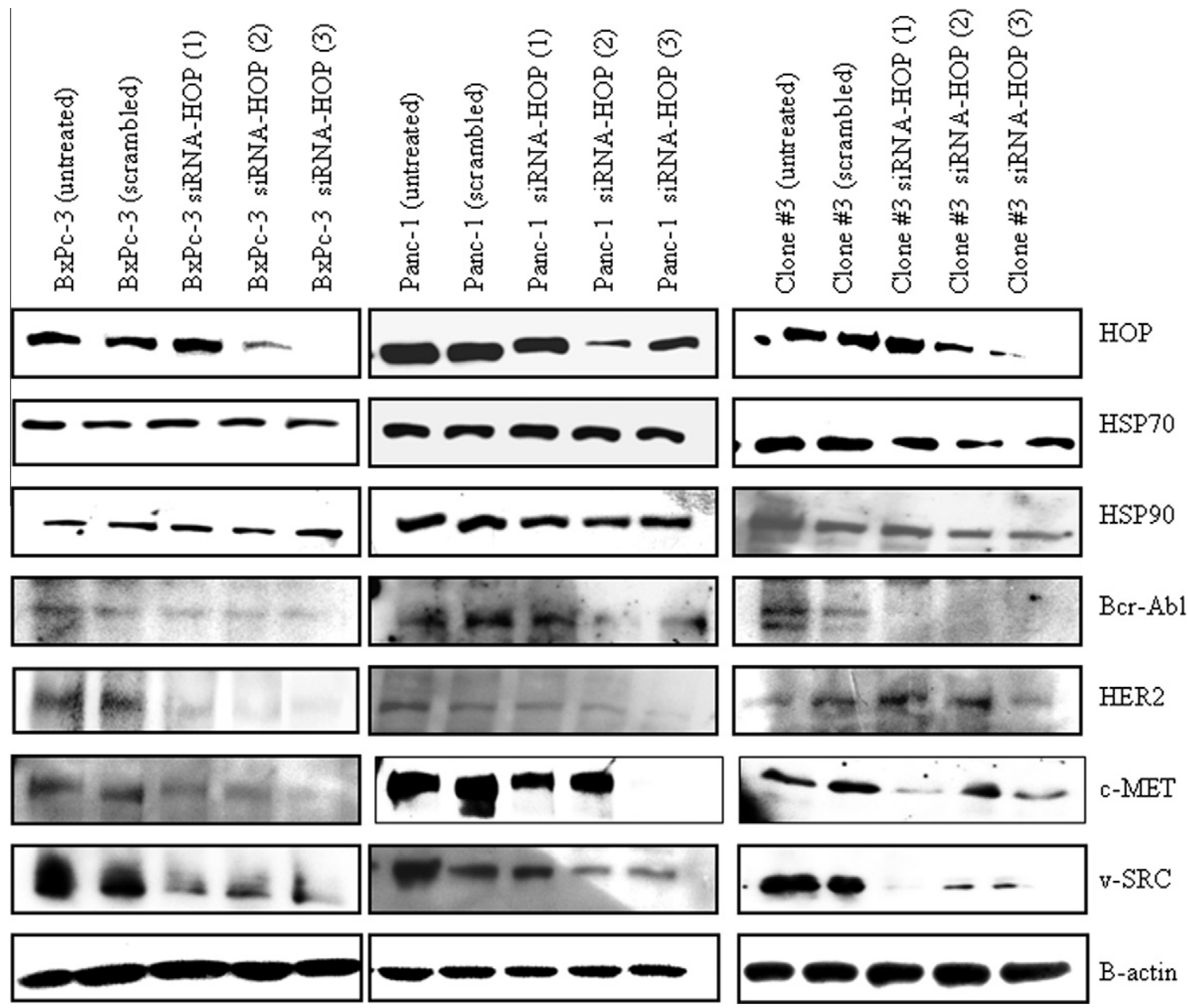

Fig. 5. Effect of Hop knockdown by siRNA on Hsp90 client proteins in BxPc-3, Panc-1 and Clone \#3. Pancreatic cancer cells were transfected with three independent siRNAs against Hop and the scrambled control siRNA. A 72 h post transfection, the constitutive levels of Hop, Hsp90, Hsp70, HER2, Bcr-Abl, cMET and v-Src were assayed by Western immunoblot.

\section{Discussion}

Previously, protein identification by 2D DIGE followed by MALDI-TOF MS established a 2-fold increase in Hop expression in the more invasive pancreatic cancer cell line Clone \#3 compared to Clone \#8 (low invasion) [17]. In this study, we investigate the role of Hop in cancer cell invasion and its potential as a therapeutic target; we firstly provide evidence that Hop expression in pancreatic cancer cell lines corresponds to their invasive abilities. We show that Hop is involved in in vitro invasion in pancreatic cancer cell lines possibly through interaction with MMP-2. To our knowledge this is the first study implicating Hop in pancreatic cancer invasion via MMP-2. Eustace et al. [21] showed that Hsp90 $\alpha$ chaperone-complex interactions are involved in MMP-2 activity and invasiveness in the fibrosarcoma cell line, HT-1080. Our work showed that loss of Hop after siRNA transfection reduced the invasiveness of BxPc-3 and Panc-1 cells; this decrease in invasion was mediated by a reduction in MMP-2 expression. The degradation of the extracellular matrix and connective tissue, leading to tumour invasion and metastasis, is facilitated by MMPs [25]. MMPs are a family of proteinase enzymes requiring a zinc ion at their active site. MMPs are secreted extracellularly in a latent form and require activation for proteolytic activity. They are inhibited by specific tissue inhibitors of metalloproteinases (TIMPs) (reviewed in [26]). Gress et al. [27,28] found that MMP-2 and MMP-9 may be involved in processes leading to the strong desmoplastic reaction - the deregulation of the extracellular matrix, including degradation and accumulation of dense fibrous tissue observed in pancreatic tumours. Hop was found in the $\mathrm{CM}$ of BxPc-3 and Panc-1 suggesting that it may interact with MMP-2 extracellularly, also defined in other studies [21]. We showed further evidence for this interaction as MMP-2 co-immunoprecipitated with Hop in BxPc-3 CM. Hsp90 also co-immunoprecipitated with Hop. Hsp90 expression in malignant cells has been reported to be constitutive, and intracellular levels are 210-fold higher compared to normal cells, suggesting a crucial role in survival and growth of cancer cells [29]. Using the specific Hsp90 inhibitor, 17-AAG, we confirmed that 

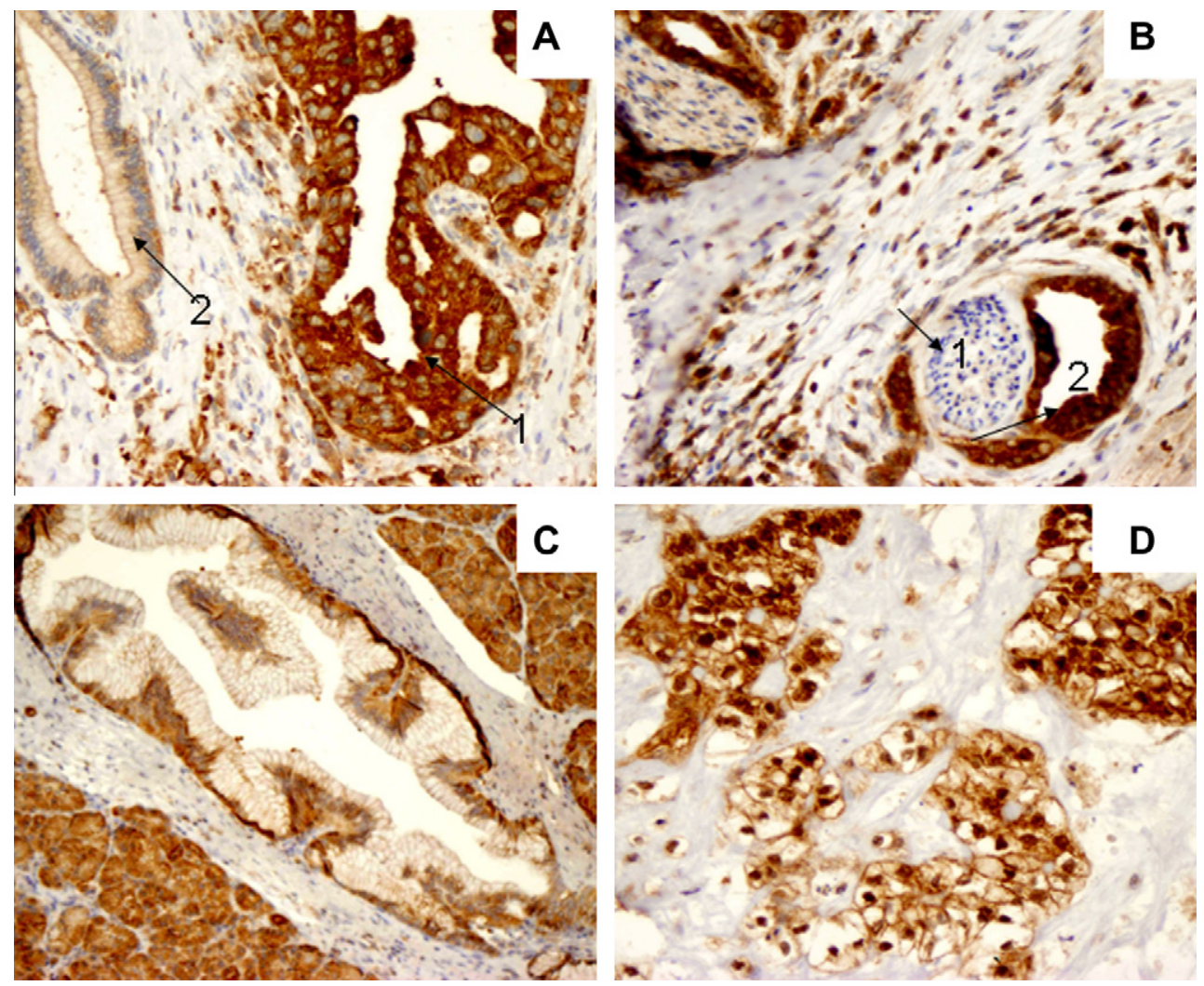

Fig. 6. Differential localisation of Hop in invasive pancreatic tumours. (A) PanIN grade 3 (arrow 1 ) displaying strong cytoplasmic Hop staining compared to PanIN grade 1 showing no Hop expression (arrow 2). (B) Perineural invasion in PDAC, cancer cells invading around the nerve (arrow 1 ) stained strongly for Hop (arrow 2). (C) PanIN grade 1 PDAC displaying sub-nuclear accumulation of Hop. (D) Invasive mucinous tumour displaying strong Hop complete membrane staining magnification $200 \times$.

inhibition of Hsp90 also decreased invasion via a decrease in MMP-2 and MMP-9 activation. This suggests a role for Hop in Hsp90-mediated inhibition of invasion through extracellular interactions. Hop acts as a co-chaperone in the Hsp90 chaperone cycle stabilising and activating a wide array of client proteins [30]. Having demonstrated that Hop knockdown resulted in decreased invasion through extracellular interaction with Hsp90 and MMP-2, we investigated whether siRNA knockdown of Hop could affect Hsp90-mediated client protein expression. Our study showed that Hop-siRNA mediated a decrease in levels of Hsp90 'client' proteins; HER2, Bcr-Abl, c-MET and $\mathrm{v}$-Src in pancreatic cancer cell lines. However, an important factor in the Hop-mediated Hsp90 inactivation could rely on whether the two proteins are complexed in the tumour cells. Kamal et al. [31] determined that Hsp90 in tumour cells is present in multi-chaperone complexes and is engaged in active chaperoning. Co-immunoprecipitation of normal and tumour cell lysates with antibodies to Hsp90 revealed that more Hsp90 in tumour cells was present in complexes with co-chaperone proteins such as p23 and Hop compared to normal cells. Immunoprecipitation with antibodies to both p23 and Hop showed that the entire tumour cell pool of Hsp90 was present in complexes unlike normal cells. These results suggest that Hsp90 in tumour cells is in the form of multi-chaperone complexes which are actively engaged in chaperoning client oncoproteins. Therefore, Hop expression, through specific inhibition of the Hsp90-Hop complexes, survival pathways and MMP activation, could contribute to invasion in the pancreatic cancer cell lines, therefore making it a potentially valuable target for pancreatic cancer therapy.

We cannot exclude the possibility that alteration of the Hsp90 pathway decreases invasion through interference with oncogenic signalling pathways. MET, a receptor for hepatoctye growth factor/scatter factor (HGF/SF) has been previously shown to play an important role in cancer invasion and metastasis [32]. As c-MET is a client of Hsp90, it is destabilised by the Hsp90 inhibitor, 17-AAG, which blocks reactivation of downstream signalling pathways (Akt Erk and/or STAT3) and EGFR family members [33]. Webb et al. [34] showed that geldanamycin (GA) down regulates MET expression at nanamolar concentrations, thus inhibiting HGF/SF-mediated cell motility and invasion. Another Hsp90 client protein, HER2 has been proposed to interact extracellularly with Hsp90 - activating HER2 by heterodimerisation with HER3, leading to actin remodelling and cell motility via activation of signal transduction pathways MAPK and PI3 K-Akt [35,36].

The localisation of Hop in pancreatic cancer tumours and its secretion by cell lines may be of importance, as shown in other studies $[21,37,9]$. The mechanism of 
secreted Hop is poorly understood. Hop is secreted from fibrosarcoma cells [21] and from primary astrocyte cultures [9,37]. Although Hop has been previously detected in the Golgi apparatus and small vesicles [38]; no transmembrane/signal peptide is contained within the Hop sequence. Therefore secretion of Hop may be through non-classical secretory routes or through its membrane interactions with prion proteins [39]. To exclude the possibility of Hop expression in the media due to cell lysis at a low rate, recombinant Hop could be used to mimic extracellular secreted Hop and assess its function extracellularly. There is evidence to suggest that localisation of Hop may be due to phosphorylation. Hop can be phosphorylated by cell cycle kinases, phosphorylation by cdc2 (cell division cycle 2) kinase promotes cytoplasmic retention, whereas phosphorylation by casein kinase II (CKII) promotes nuclear Hop, suggesting that Hop might move between the cytoplasm and the nucleus under certain cell cycle conditions [40,41]. The nuclear accumulation of Hop is primarily stress regulated and CKII phosphorylation has been shown not to disrupt Hop-Hsp90 binding [36]. Longshaw et al. [42] reported that in the absence of Hop, soluble pYStat3 levels are decreased and accumulation of Stat3 occurs outside the nucleus in mESCs, suggesting that Hop facilitates phosphorylation and transport of Stat3 into the mESCs nucleus.

Immunohistochemical analysis revealed a correlation between Hop expression and the degree of differentiation in PDAC tumours. The PanIN lesions show sequential changes in Hop expression levels from strong staining in high PanIN grades and invasive carcinoma compared to weak Hop staining in low PanIN grades. Differential Hop expression occurs at different stages of the malignant progression of PDAC, suggesting a progressive increase in expression as the tumour becomes more malignant, possibly due to localisation of Hop resulting in nuclear/cytoplasmic or membrane accumulation of the protein.

In summary, we have demonstrated for the first time that siRNAs specifically targeting human Hop substantially reduced the invasion of human pancreatic cancer cells in vitro through extracellular interactions with MMP-2 and Hsp90. Decreasing Hop levels by siRNA also degraded Hsp90 client proteins, HER2, Bcr-Abl, c-MET and v-Src possibly through Hsp90 inactivation. Targeting the Hsp90 pathway through Hop is an attractive strategy as multiple oncoproteins are dependent on this chaperone activity. Our results suggest that disruption of the Hop-Hsp90 complex may provide a novel mechanism for Hsp90 dependent pathways in pancreatic cancer. Recently, Horibe et al. [43] designed a novel peptide which disrupts the interaction between Hsp90 and the TPR2A binding domain of Hop. This specific inhibitor of Hsp90-Hop interaction showed selective antitumour activity, discriminating between normal and cancerous cell lines. Cells treated with the inhibitor exhibited loss of Hsp90-client proteins, survivin, CDK4 and Akt. Antitumour activity was also observed in vivo in a xenograft model of human pancreatic cancer in mice.

The differential localisation of Hop in invasive pancreatic tumours is interesting and further work into the significance of this observation is required in a larger panel of pancreatic cancer specimens and additional confocal analysis in cell lines.

\section{Conflict of interest statement}

The authors declare no conflict of interest.

\section{Acknowledgments}

The authors wish to thank the Irish Higher Education Authority, Program of Research in Third Level Institutions (PRTLI Cycle III and IV) and the Science Foundation Ireland (SFI) for funding this work.

\section{References}

[1] A. Jemal, R. Siegel, E. Ward, T. Murray, J. Xu, M.J. Thun, Cancer statistics, CA Cancer J. Clin. 57 (2007) 43-66.

[2] M.F. Brennan, Adjuvant therapy following resection for pancreatic adenocarcinoma, Surg. Oncol. Clin. N. Am. 13 (2004) 555-566. vii.

[3] P.E. Carrigan, L.A. Sikkink, D.F. Smith, M. Ramirez-Alvarado, Domain: domain interactions within hop, the Hsp70/Hsp90 organizing protein, are required for protein stability and structure, Protein Sci. 15 (2006) 522-532.

[4] S. Chen, D.F. Smith, Hop as an adaptor in the heat shock protein 70 (Hsp70) and hsp90 chaperone machinery, J. Biol. Chem. 273 (1998) 35194-35200.

[5] M.P. Hernandez, W.P. Sullivan, D.O. Toft, The assembly and intermolecular properties of the hsp70-Hop-hsp90 molecular chaperone complex, J. Biol. Chem. 277 (2002) 38294-38304.

[6] H. Wegele, L. Muller, J. Buchner, Hsp70 and Hsp90 - a relay team for protein folding, Rev. Physiol. Biochem. Pharmacol. 151 (2004) 1-44.

[7] H. Kubota, S. Yamamoto, E. Itoh, et al., Increased expression of cochaperone HOP with HSP90 and HSC70 and complex formation in human colonic carcinoma, Cell Stress Chaperones. 15 (2010) 10031011.

[8] W. Sun, B. Xing, Y. Sun, et al., Proteome analysis of hepatocellular carcinoma by two-dimensional difference gel electrophoresis: novel protein markers in hepatocellular carcinoma tissues, Mol. Cell. Proteomics. 6 (2007) 1798-1808.

[9] R.B. Erlich, S.A. Kahn, F.R. Lima, A.G. Muras, R.A. Martins, R. Linden, L.B. Chiarini, V.R. Martins, V. Moura Neto, STI1 promotes glioma proliferation through MAPK and PI3K pathways, Glia 55 (2007) 1690-1698.

[10] A.J. Bredemeyer, P.E. Carrigan, T.A. Fehniger, D.F. Smith, T.J. Ley, Hop cleavage and function in granzyme B-induced apoptosis, J. Biol. Chem. 281 (2006) 37130-37141.

[11] E. Pick, Y. Kluger, J.M. Giltnane, C. Moeder, R.L. Camp, D.L. Rimm, H.M. Kluger, High HSP90 expression is associated with decreased survival in breast cancer, Cancer Res. 67 (2007) 2932-2937.

[12] M. Ogata, Z. Naito, S. Tanaka, Y. Moriyama, G. Asano, Overexpression and localization of heat shock proteins mRNA in pancreatic carcinoma, J. Nippon Med. Sch. 67 (2000) 177-185.

[13] A. Aghdassi, P. Phillips, V. Dudeja, D. Dhaulakhandi, R. Sharif, R. Dawra, M.M. Lerch, A. Saluja, Heat shock protein 70 increases tumorigenicity and inhibits apoptosis in pancreatic adenocarcinoma, Cancer Res. 67 (2007) 616-625.

[14] S. Lindquist, The heat-shock response, Annu. Rev. Biochem. 55 (1986) 1151-1191.

[15] S.A. Fuqua, S. Oesterreich, S.G. Hilsenbeck, D.D. Von Hoff, J. Eckardt, C.K. Osborne, Heat shock proteins and drug resistance, Breast Cancer Res. Treat. 32 (1994) 67-71.

[16] M.V. Powers, P. Workman, Targeting of multiple signalling pathways by heat shock protein 90 molecular chaperone inhibitors, Endocr. Relat. Cancer. 13 (Suppl. 1) (2006) S125-35.

[17] N. Walsh, N. O’Donovan, S. Kennedy, M. Henry, P. Meleady, M. Clynes, P. Dowling, Identification of pancreatic cancer invasionrelated proteins by proteomic analysis, Proteome Sci. 7 (2009) 3.

[18] N. Walsh, M. Clynes, J. Crown, N. O’Donovan, Alterations in integrin expression modulates invasion of pancreatic cancer cells, J. Exp. Clin. Cancer Res. 28 (2009) 140.

[19] A. Albini, Y. Iwamoto, H.K. Kleinman, G.R. Martin, S.A. Aaronson, J.M. Kozlowski, R.N. McEwan, A rapid in vitro assay for quantitating the invasive potential of tumor cells, Cancer Res. 47 (1987) 3239-3245 
[20] B.K. Eustace, T. Sakurai, J.K. Stewart, et al., Functional proteomic screens reveal an essential extracellular role for hsp90 alpha in cancer cell invasiveness, Nat. Cell Biol. 6 (2004) 507-514.

[21] B.K. Eustace, D.G. Jay, Extracellular roles for the molecular chaperone, hsp90, Cell Cycle 3 (2004) 1098-1100.

[22] S. Tsutsumi, B. Scroggins, F. Koga, M.J. Lee, J. Trepel, S. Felts, C. Carreras, L. Neckers, A small molecule cell-impermeant Hsp90 antagonist inhibits tumor cell motility and invasion, Oncogene 27 (2008) 2478-2487.

[23] D. Stellas, A. Karameris, E. Patsavoudi, Monoclonal antibody 4C5 immunostains human melanomas and inhibits melanoma cell invasion and metastasis, Clin. Cancer Res. 13 (2007) 1831-1838.

[24] M.S. Kim, H.J. Kwak, J.W. Lee, et al., 17-Allylamino-17demethoxygeldanamycin down-regulates hyaluronic acid-induced glioma invasion by blocking matrix metalloproteinase- 9 secretion, Mol. Cancer. Res. 6 (2008) 1657-1665.

[25] D.E. Kleiner, W.G. Stetler-Stevenson, Matrix metalloproteinases and metastasis, Cancer Chemother. Pharmacol. 43 (1999) S42-51. Suppl.

[26] H.D. Foda, S. Zucker, Matrix metalloproteinases in cancer invasion, metastasis and angiogenesis, Drug Discov. Today 6 (2001) 478482.

[27] T.M. Gress, F. Muller-Pillasch, M.M. Lerch, H. Friess, M. Buchler, G. Adler, Expression and in-situ localization of genes coding for extracellular matrix proteins and extracellular matrix degrading proteases in pancreatic cancer, Int. J. Cancer 62 (1995) 407-413.

[28] T.M. Gress, F. Muller-Pillasch, C. Weber, M.M. Lerch, H. Friess, M Buchler, H.G. Beger, G. Adler, Differential expression of heat shock proteins in pancreatic carcinoma, Cancer Res. 54 (1994) 547-551.

[29] M. Ferrarini, S. Heltai, M.R. Zocchi, C. Rugarli, Unusual expression and localization of heat-shock proteins in human tumor cells, Int. J. Cancer 51 (1992) 613-619.

[30] M.A. Brown, L. Zhu, C. Schmidt, P.W. Tucker, Hsp90-from signal transduction to cell transformation, Biochem. Biophys. Res. Commun. 363 (2007) 241-246.

[31] A. Kamal, L. Thao, J. Sensintaffar, L. Zhang, M.F. Boehm, L.C. Fritz, F.J. Burrows, A high-affinity conformation of Hsp90 confers tumour selectivity on Hsp90 inhibitors, Nature 425 (2003) 407-410.

[32] C. Birchmeier, W. Birchmeier, E. Gherardi, G.F. Vande Woude, Met, metastasis, motility and more, Nat. Rev. Mol. Cell Biol. 4 (2003) 915925
[33] S. Wang, I. Pashtan, S. Tsutsumi, W. Xu, L. Neckers, Cancer cells harboring MET gene amplification activate alternative signaling pathways to escape MET inhibition but remain sensitive to Hsp90 inhibitors, Cell Cycle 8 (2009) 2050-2056.

[34] C.P. Webb, C.D. Hose, S. Koochekpour, M. Jeffers, M. Oskarsson, E. Sausville, A. Monks, G.F. Vande Woude, The geldanamycins are potent inhibitors of the hepatocyte growth factor/scatter factormet-urokinase plasminogen activator-plasmin proteolytic network, Cancer Res. 60 (2000) 342-349.

[35] K. Sidera, M. Gaitanou, D. Stellas, R. Matsas, E. Patsavoudi, A critical role for HSP90 in cancer cell invasion involves interaction with the extracellular domain of HER-2, J. Biol. Chem. 283 (2008) 2031-2041.

[36] K. Sidera, E. Patsavoudi, Extracellular HSP90: conquering the cell surface, Cell Cycle 7 (2008) 1564-1568.

[37] F.R. Lima, C.P. Arantes, A.G. Muras, R. Nomizo, R.R. Brentani, V.R. Martins, Cellular prion protein expression in astrocytes modulates neuronal survival and differentiation, J. Neurochem. 103 (2007) 2164-2176.

[38] B. Honore, H. Leffers, P. Madsen, H.H. Rasmussen, J. Vandekerckhove, J.E. Celis, Molecular cloning and expression of a transformationsensitive human protein containing the TPR motif and sharing identity to the stress-inducible yeast protein STI1, J. Biol. Chem. 267 (1992) 8485-8491.

[39] S.M. Zanata, M.H. Lopes, A.F. Mercadante, et al., Stress-inducible protein 1 is a cell surface ligand for cellular prion that triggers neuroprotection, EMBO J. 21 (2002) 3307-3316.

[40] V.M. Longshaw, J.P. Chapple, M.S. Balda, M.E. Cheetham, G.L. Blatch, Nuclear translocation of the Hsp70/Hsp90 organizing protein mSTI1 is regulated by cell cycle kinases, J. Cell Sci. 117 (2004) 701-710.

[41] S. Daniel, G. Bradley, V.M. Longshaw, C. Soti, P. Csermely, G.L. Blatch, Nuclear translocation of the phosphoprotein hop (Hsp70/Hsp90 organizing protein) occurs under heat shock and its proposed nuclear localization signal is involved in Hsp90 binding, Biochim. Biophys. Acta 1783 (2008) 1003-1014.

[42] V.M. Longshaw, M. Baxter, M. Prewitz, G.L. Blatch, Knockdown of the co-chaperone eu promotes extranuclear accumulation of Stat3 in mouse embryonic stem cells, Eur. J. Cell Biol. 88 (2009) 153-166.

[43] T. Horibe, M. Kohno, M. Haramoto, K. Ohara, K. Kawakami, Designed hybrid TPR peptide targeting Hsp90 as a novel anticancer agent, J. Trans. Med. 9 (2011) 8. 